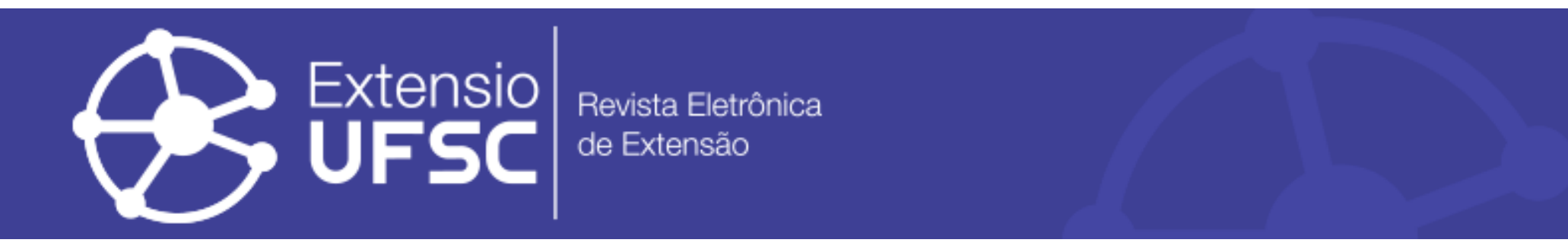

\title{
TECNOLOGIAS DESENVOLVIDAS PELAS INSTITUIÇÕES DE ENSINO SUPERIOR PARA IDOSOS COM DEFICIÊNNCIA
}

\author{
Hundra Prestes de Godoi \\ Universidade Federal de Santa Catarina \\ hundrapg@gmail.com \\ Juliana Balbinot Reis Girondi \\ Universidade Federal de Santa Catarina \\ juliana.balbinot@ufsc.br \\ Jordelina Schier \\ Universidade Federal de Santa Catarina \\ jordelina.schier@ufsc.br
}

\author{
Darla Lusia Ropelato Fernandez \\ Universidade Federal de Santa Catarina \\ darlaropelato@gmail.com \\ Francisco Reis Tristão \\ Universidade Federal de Santa Catarina \\ franciscoreistristao@hotmail.com \\ Fernanda Rosa de Oliveira Pires \\ Universidade Federal de Santa Catarina \\ nandadode@hotmail.com
}

\begin{abstract}
Resumo
Introdução: Com o aumento do envelhecimento populacional e das Doenças Crônicas é cada vez mais prevalente pessoas idosas com deficiência. Consoante, as instituições formadoras na área da saúde devem preparar os futuros profissionais para estas demandas desenvolvendo oportunidades de aprendizado e novas tecnologias de cuidado. Objetivo: investigar as tecnologias de cuidado ao idoso com deficiência nas Instituições de Ensino Superior (IES) que oferecem cursos na área da saúde na região da Grande Florianópolis/Santa Catarina. Método: pesquisa mista exploratório-descritiva realizada com 26 coordenadores de cursos de saúde das IES da Grande Florianópolis/ de novembro/2016 a abril/2017. Os dados foram coletados mediante questionário estruturado e submetidos à análise temática de conteúdo de Minayo. Os resultados foram discutidos à luz da literatura pertinente. Resultados: dos atendimentos realizados, $86 \%$ dos cursos o desenvolvem somente para idosos e 59\% para idoso com deficiência. Quanto aos projetos de pesquisa e extensão 72,7\% dos cursos realizam projetos para pessoas idosas e 50\% especificamente para idosos com deficiência. No quesito atividades de lazer, $23 \%$ dos coordenadores responderam que as realizam para o público idoso e 18\% ao idoso com deficiência. Conclusões: a maioria dos cursos de graduação na área da saúde desenvolve tecnologias de cuidado relacionadas ao envelhecimento e deficiências, mas relacioná-los ao currículo, pesquisa e extensão ainda são incipientes, considerando a problemática populacional emergente no Brasil.
\end{abstract}

Palavras-chave: Idoso. Pessoas com Deficiência. Tecnologia em Saúde.

\section{TECHNOLOGIES DEVELOPED BY HIGHER EDUCATION INSTITUTIONS FOR ELDERLY DISABLED PEOPLE}

\begin{abstract}
Introduction: Older people with disabilities are gradually prevalent, with increasing population aging and chronic diseases, Accordingly, health education institutions should prepare future professionals for these demands by developing learning opportunities and new technologies of care. Objective: to investigate the technologies of elderly care with disabilities in Higher Education Institutions that offer courses in health care in the region of "Florianópolis / SC". Methodology: a mixed exploratory-descriptive research carried out with 26 coordinators of IES courses in "Grande Florianópolis" / from November / 2016 to April / 2017. Data were collected through a structured questionnaire and submitted to Minayo content thematic analysis. The results were discussed through relevant literature. Results: $86 \%$ of the courses offered are for the elderly and $59 \%$ for the elderly with disabilities. As for the research and extension projects, $72.7 \%$ of the courses carry out projects for the elderly and 50\% specifically for the elderly with disabilities. In terms of leisure activities, $23 \%$ of the coordinators answered that they are doing it for the elderly and 18\% for the elderly with disabilities. Conclusions: most of the undergraduate courses in the health area develop care technologies related to aging and disabilities, but relate them to the curriculum, research and extension are still incipient, considering the emerging population problem in Brazil. Keywords: Elderly. Disabled People. Health's Technology.
\end{abstract}




\section{TECNOLOGÍAS DESARROLLADAS POR LAS INSTITUCIONES DE ENSEÑANZA SUPERIOR PARA AÑOS CON DISCAPACIDAD}

\section{Resumen}

Introducción: Con el crecimiento del envejecimiento poblacional y de las enfermedades crónicas es cada vez más común que hayan personas mayores con discapacidad. Además, las universidades y escuelas que tienen cursos en el área de la salud deben preparar a los futuros profesionales para estas demandas desarrollando oportunidades de aprendizaje y nuevas tecnologías de cuidado. Objetivo: investigar las tecnologías de cuidado al anciano con discapacidad en las Instituciones de enseñanza superior (IES) que ofrecen cursos en el área de la salud en la región de la Grande Florianópolis / SC. Metodología: investigación mixta exploratoria-descriptiva realizada con 26 coordinadores de cursos de salud de las IES de la Grande Florianópolis / de noviembre / 2016 a abril / 2017. Los datos fueron recolectados mediante cuestionario estructurado y sometidos al análisis temático de contenido de Minayo. Los resultados se discutieron con base en la literatura relacionada. Resultados: de las atenciones realizadas $86 \%$ de los cursos lo desarrollan solamente para ancianos y $59 \%$ para ancianos con discapacidad. Los proyectos de investigación y extensión el 72,7\% de los cursos realizan proyectos para personas mayores y $50 \%$ específicamente para ancianos con discapacidad. En relacion a las actividades de ocio el $23 \%$ de los coordinadores respondieron que las realizan para el público anciano y el $18 \%$ al anciano con discapacidad. Conclusiones: la mayoría de los cursos de graduación en el área de la salud desarrollan tecnologías de cuidado relacionadas con el envejecimiento y deficiencias, pero relacionarlos al currículo, investigación y extensión todavía son insuficientes, considerando la problemática poblacional emergente en Brasil.

Palavras clave: Ancianos. Personas con Deficiencia. Tecnología en Salud. 
Tecnologias desenvolvidas pelas instituições de ensino superior para idosos com deficiência

\section{INTRODUÇÃO}

Frente ao envelhecimento populacional que vem ocorrendo nos últimos anos, o Brasil adotou políticas voltadas a esse público, a exemplo da Política Nacional do Idoso (1994), o Estatuto do Idoso (2003) e a Política Nacional de Saúde da Pessoa Idosa (2006), que preveem a garantia da atenção integral à saúde da população idosa no Sistema Único de Saúde (SUS), incentivam a pesquisa, a adequação de currículos, metodologias e material didático de formação de profissionais na área da saúde, bem como priorizam o envelhecimento saudável, familiar e ativo, oportunizando o protagonismo e autonomia dos idosos no Brasil. (GIRONDI et. al, 2013).

Paralelamente ao envelhecimento populacional, percebe-se também o crescimento na prevalência de deficiências que, na população idosa, tem aumentado em consequência do crescimento das Doenças Crônicas Não Transmissíveis (DCNT), associadas a um envelhecer senil. Assim, políticas existentes necessitam de reformas estruturais de modo que a população idosa com deficiência também tenha garantido atendimento em saúde e tenha direitos reservados com respeito ao acesso e investimento em tecnologias do cuidado (RESENDE; NERI, 2011).

Em 2010, já se estimava que $14,5 \%$ da população brasileira tinha algum tipo de deficiência e, entre as pessoas identificadas, a maioria eram pessoas idosas com deficiência física, visual, auditiva e importantes lesões incapacitantes, tornando este público vulnerável à dependência e com risco de perda da autonomia. Entre as principais causas que levaram estas pessoas a tornarem-se deficientes destacam-se as DCNT e o seu não tratamento e/ou tratamento inadequado (BRASIL, 2010a; 2010b).

Diante da importância do assunto, algumas iniciativas governamentais foram tomadas a partir de 2011, como o Plano Nacional dos Direitos da Pessoa com Deficiência e as Portarias MS/GM 793/2012 e 835/2012 que implementaram a Rede de Cuidados à Pessoa Com Deficiência no âmbito do SUS (BRASIL, 2012a; 2017).

Visto que a rede de saúde destinada ao idoso e a pessoa com deficiência busca qualidade de vida para este público, é relevante que a formação de futuros profissionais de saúde busque qualificação, desenvolva e reconheça tecnologias de cuidado que auxiliem na assistência a estas pessoas. Considerando que a região da Grande Florianópolis-SC apresenta grande potencial para exercer ações voltadas ao público em questão, uma vez que podem contar com subsídios e expertise científica, torna-se importante conhecer quais são as tecnologias inovadoras que tem sido implementadas para promover a saúde das pessoas idosas com deficiência por estas instituições. 
Tecnologias desenvolvidas pelas instituições de ensino superior para idosos com deficiência

Diante do exposto, essa pesquisa tem como objetivo investigar as tecnologias de cuidado ao idoso com deficiência nas IES que oferecem cursos na área da saúde da região elencada.

\section{MATERIAIS E MÉTODOS}

Trata-se de uma pesquisa realizada entre novembro de 2016 a abril de 2017, com Coordenadores de Cursos da Saúde nas IES da região da Grande Florianópolis/SC.

Os participantes foram convidados a participar por meio de correio eletrônico, contato telefônico ou pessoalmente. Para a coleta de dados foi aplicado questionário estruturado preenchido pelos participantes via Internet (Google Docs $\left.{ }^{\circledR}\right)$ para caracterizar os coordenadores bem como cada curso, especialmente no que tange o oferecimento de algum tipo de atendimento de saúde ao idoso e/ou idoso com deficiência, qual fluxo de acesso, tipo de deficiência atendida, se existia algum projeto de pesquisa e extensão com pessoas idosas e idosos com deficiência, assim como quais as tecnologias inovadoras do cuidado eram proporcionadas pelos cursos para pessoas idosas e idosos com deficiência.

Não foram atribuídos critérios de inclusão e exclusão. Os participantes excluídos se deram devido a condições de respeito a não aceitação em participar do trabalho e por falta de resposta ao questionário em tempo hábil de coleta de dados. Assim, foram convidados 26 coordenadores sendo que quatro foram excluídos por não responder em tempo hábil. Para garantir o anonimato eles foram identificados no estudo por C1, C2, sucessivamente até C22.

Para análise dos dados qualitativos utilizou-se a Análise Temática de Minayo (2015). Esta pesquisa está vinculada ao macroprojeto "A rede de atenção à saúde e de suporte social à pessoa idosa com deficiência na Grande Florianópolis e as tecnologias de cuidado" aprovado pelo Comitê de ética com parecer no 24410513.5.0000.0121.

\section{RESULTADOS}

Conforme representado no quadro 1, dentre os 22 Coordenadores dos cursos da saúde nas IES na Grande Florianópolis/SC participantes desta pesquisa, 40,9\% são procedentes de IES pública federal, 4,5\% de IES pública estadual e 54,5\% de IES privadas. O perfil dos participantes é composto por 12 mulheres (55\%) e 10 homens (45\%). No quesito idade, a faixa etária prevalente é a de 40 a 49 anos, representando 41\% (9) dos participantes, seguida da faixa entre 50 a $59 \operatorname{anos}(32 \%)$, e $23 \%$ entre 30 e 39 anos. Somente um participante informou idade acima de 60 $\operatorname{anos}(5 \%)$. 
Tecnologias desenvolvidas pelas instituições de ensino superior para idosos com deficiência

No quesito formação, observou-se que a coordenação do curso em 100\% é realizada pelo profissional correspondente e todos os coordenadores são titulados em pós-graduação (59\% doutorado, $32 \%$ mestrado, $5 \%$ com pós-doutorado e 5\% com especialização).

Quadro 1 - Perfil dos coordenadores de vinte e dois cursos de graduação na área da saúde em IES da Grande Florianópolis/SC, 2017.

\begin{tabular}{|c|c|c|c|c|c|}
\hline Coordenador & Sexo & Idade & Formação & $\begin{array}{c}\text { Possui } \\
\text { Pós } \\
\text { Graduação }\end{array}$ & $\begin{array}{l}\text { Tipo de Pós } \\
\text { Graduação }\end{array}$ \\
\hline C1 & $\mathrm{F}$ & 53 & $\begin{array}{l}\text { Professora de Educação } \\
\text { Física e Fonoaudióloga }\end{array}$ & SIM & Doutorado \\
\hline $\mathrm{C} 2$ & $\mathrm{M}$ & 32 & Naturologia Aplicada & SIM & Mestrado \\
\hline C3 & M & 52 & $\begin{array}{l}\text { Licenciado em } \\
\text { Educação Física }\end{array}$ & SIM & Doutorado \\
\hline $\mathrm{C} 4$ & $\mathrm{~F}$ & 38 & Fonoaudióloga & SIM & Doutorado \\
\hline $\mathrm{C} 5$ & $\mathrm{~F}$ & 41 & Fisioterapeuta & SIM & Mestrado \\
\hline C6 & $\mathrm{F}$ & 31 & Educação Física & SIM & Mestrado \\
\hline $\mathrm{C} 7$ & $\mathrm{M}$ & 45 & Psicologia & SIM & Mestrado \\
\hline $\mathrm{C} 8$ & $\mathrm{~F}$ & 54 & Odontologia & SIM & Pós-doutorado \\
\hline C9 & $\mathrm{M}$ & 53 & Enfermeiro & SIM & Mestrado \\
\hline $\mathrm{C} 10$ & $\mathrm{M}$ & 35 & Fisioterapia & SIM & Mestrado \\
\hline C11 & M & 44 & $\begin{array}{c}\text { Farmacêutico - } \\
\text { Bioquímico }\end{array}$ & SIM & Doutorado \\
\hline $\mathrm{C} 12$ & $\mathrm{M}$ & 58 & Psicologia & SIM & Doutorado \\
\hline C13 & $\mathrm{F}$ & 41 & Nutrição & SIM & Mestrado \\
\hline $\mathrm{C} 14$ & $\mathrm{~F}$ & 41 & Psicologia & SIM & Doutorado \\
\hline C15 & $\mathrm{F}$ & 53 & Odontologia & SIM & Doutorado \\
\hline C16 & $\mathrm{M}$ & 68 & Médico & SIM & Especialização \\
\hline $\mathrm{C} 17$ & $\mathrm{~F}$ & 57 & Nutrição & SIM & Doutorado \\
\hline C18 & $\mathrm{F}$ & 38 & Farmacêutica & SIM & Doutorado \\
\hline C19 & $\mathrm{F}$ & 47 & Educação Física & SIM & Doutorado \\
\hline $\mathrm{C} 20$ & $\mathrm{M}$ & 40 & Enfermeiro & SIM & Doutorado \\
\hline C21 & $\mathrm{M}$ & 40 & Médico & SIM & Doutorado \\
\hline $\mathrm{C} 22$ & $\mathrm{~F}$ & 49 & Serviço Social & SIM & Doutorado \\
\hline
\end{tabular}

Fonte: Dados fornecidos pelos coordenadores de IES públicas e privadas, elaborado pelos autores, 2017.

\section{Tipo de atendimento oferecido ao público idoso e idoso com deficiência}

Entre os 22 cursos de IES participantes, $86 \%$ oferecem algum tipo de atendimento ao idoso e 59\% ao idoso com deficiência, incluindo educação em saúde, avaliação clínica individual, grupos de saúde e grupos de idosos. O fluxo e acesso a tais atendimentos ocorrem, em sua maioria, nas UBS, Instituição de Longa Permanência de Idosos (ILPI), Centro de Atenção a Terceira Idade (CATI), de consulta ambulatorial/hospitalar e Clínica Escola. 
Dos atendimentos realizados pelas IES, 86\% são dirigidos ao idoso, 41\% ocorrem através de estágios supervisionados na Atenção Básica ou Área Hospitalar. Os coordenadores C3, C9 e C20 destacam que são desenvolvidas ações educativas por meio das disciplinas optativas do curso, as quais estudam a fisiologia do envelhecimento e alterações orgânicas, envelhecimento saudável e principais patologias do idoso.

O coordenador C5 descreve que o curso oferece atendimento para idosos com disfunções ortofuncionais, neurofuncionais e respiratórias, bem como fisioterapia aquática para idosos da Clínica de Fisioterapia da IES. O atendimento se dá por demanda espontânea e o acesso é feito por meio de contato telefônico e lista de espera. Já o coordenador C7, descreve avaliação neuropsicológica e psicoterapia para idosos mediante inscrição na clínica escola da instituição.

No que se refere ao atendimento do idoso com deficiência nas IES, 59\% dos cursos da área de saúde desenvolvem atividades para esse público, sendo que destes, 76,9\% atendem idosos com deficiência múltipla, seguidas de deficiência física, auditiva e intelectual com 7,7\% cada. A faixa etária prevalente é de 60 a 65 anos (54\%), seguida de 66 a 70 anos (38\%) e apenas 8\% na faixa dos 76 aos 80 anos de idade.

O curso coordenado por C3 oferece musculação para mulheres idosas com incontinência urinária. O acesso a este serviço pode ocorrer por meio da rede de saúde municipal da região continental de Florianópolis/SC, da Rede Feminina de Combate ao Câncer da região e por meio de inscrição no grupo de estudos da terceira idade instituído por este curso. Já o coordenador C4 descreve oferecer atendimento clínico a pessoas idosas com deficiência auditiva que participam em grupos da atenção primária e de convivência. Além desses acessos, outros idosos podem buscar o serviço diretamente na clínica escola mantida pelo curso.

O coordenador C22 destaca a importância do atendimento prestado ao idoso com deficiência dentro da rede municipal assistencial, que por intermédio de ações diretas ou indiretas em instituições como Hospitais, Centro de Atenção Psicossocial (CAPS), Equipe NASF e outras equipes e entidades aliadas, os alunos de graduação têm a oportunidade de acompanhar e desenvolver atendimentos ao público idoso com deficiência.

\section{Projetos de extensão e pesquisa desenvolvidos para o público idoso e idoso com deficiência}

Dentre os 22 Coordenadores participantes desta pesquisa, 72,7\% relataram que é realizado algum projeto específico para pessoas idosas e 50\% disseram que é realizado algum projeto específico para pessoas idosas com deficiência, ilustrados no quadro 2. 
Tecnologias desenvolvidas pelas instituições de ensino superior para idosos com deficiência

Quadro 2 - Projetos desenvolvidos para pessoas idosas em vinte e dois cursos de graduação na área da saúde em IES da Grande Florianópolis/SC. 


\begin{tabular}{|c|c|c|}
\hline Coordenador & Projeto Idoso & Tipo de Projeto \\
\hline C1 & $\begin{array}{l}\text { Educação em saúde visando alcançar o conceito de } \\
\text { práticas corporais como linguagem para mudança de } \\
\text { hábitos e estilo de vida. }\end{array}$ & Não informou \\
\hline $\mathrm{C} 2$ & Atividades de promoção da saúde entre outros. & Não informou \\
\hline \multirow[b]{2}{*}{ C3 } & $\begin{array}{l}\text { Através do Grupo de Estudos da Terceira Idade são } \\
\text { desenvolvidos projetos de caminhada; dança; } \\
\text { ginástica; hidroginástica; musculação; natação; } \\
\text { pilates; Universidade Aberta; Canto; Prevenção e } \\
\text { tratamento das disfunções do assoalho pélvico. }\end{array}$ & Extensão \\
\hline & $\begin{array}{l}\text { Projetos de pesquisa desenvolvidos através do } \\
\text { Laboratório de Gerontologia: } \\
\text { Nível de Atividade Física, Rede de Suporte Social e } \\
\text { Funcionalidade Familiar de Idosos Centenários da } \\
\text { Grande Florianópolis. } \\
\text { Programa "Academia da Saúde" em Santa Catarina: } \\
\text { característica e participação de idosos. } \\
\text { Avaliação do equilíbrio e medo de cair em idosos. }\end{array}$ & Pesquisa \\
\hline C4 & $\begin{array}{l}\text { Projetos com objetivo de avaliar a audição, a } \\
\text { cognição, memória e também projetos de prevenção } \\
\text { e promoção da saúde, saúde auditiva, saúde vocal e } \\
\text { alimentar. Desenvolvidos na Clínica escola, } \\
\text { NETI/UFSC e outros grupos. }\end{array}$ & $\begin{array}{l}\text { Pesquisa e } \\
\text { Extensão }\end{array}$ \\
\hline \multirow[b]{2}{*}{ C5 } & $\begin{array}{l}\text { Cinesioterapia em pessoas com artrose de joelho, } \\
\text { com objetivo de verificar os efeitos da mesma na } \\
\text { dor e sua melhora; Inclusão digital relacionado com } \\
\text { a demência. }\end{array}$ & Pesquisa \\
\hline & $\begin{array}{l}\text { Fisioterapia aquática para idosos com osteoartrose } \\
\text { de coluna e joelho, com objetivo de minimizar a dor } \\
\text { e melhorar a função; Inclusão digital, buscando } \\
\text { estimular a memória e evitar a demência. Coração } \\
\text { saudável desenvolvendo atividade física e exercício } \\
\text { para promoção de saúde do idoso. }\end{array}$ & Extensão \\
\hline C6 & $\begin{array}{l}\text { Desenvolve projetos, no entanto não especificou.O } \\
\text { público idoso acessa o projeto pelo telefone, de } \\
\text { forma presencial e pelo site. }\end{array}$ & Extensão \\
\hline $\mathrm{C} 8$ & Saúde bucal para idosos de ILPI em Palhoça/SC. & $\begin{array}{l}\text { Pesquisa } \\
\text { Extensão }\end{array}$ \\
\hline C9 & Desenvolve projetos, no entanto não especificou. & Não informou \\
\hline C11 & Atenção Farmacêutica & Extensão \\
\hline C14 & $\begin{array}{l}\text { Programa de preparação para a aposentadoria. } \\
\text { Projetos pontuais de pesquisa que envolve o tema da } \\
\text { velhice. }\end{array}$ & $\begin{array}{l}\text { Pesquisa } \\
\text { extensão }\end{array}$ \\
\hline C15 & $\begin{array}{l}\text { Projeto de extensão com atendimento nas clínicas } \\
\text { odontológicas }\end{array}$ & Extensão \\
\hline C16 & Oficina da lembrança & Não informou \\
\hline C17 & $\begin{array}{l}\text { Educação continuada à clientes diabéticos, através } \\
\text { do ambulatório do Hospital Universitário/UFSC } \\
\text { Atendimento sistematizado em ambulatório de }\end{array}$ & Extensão \\
\hline
\end{tabular}




\begin{tabular}{|l|l|l|}
\hline & $\begin{array}{l}\text { nutrição para população de Florianópolis; } \\
\text { Nutrição na Terceira Idade (NUTI) - (no } \\
\text { NETI/Grupo de atenção) - Proposta de trabalhar } \\
\text { com estilo de vida saudável. }\end{array}$ & \\
\hline $\mathbf{C 1 8}$ & $\begin{array}{l}\text { Projeto de extensão realizado com idosos, com } \\
\text { objetivo de avaliar o perfil lipídico e o risco de } \\
\text { desenvolver doença coronariana. }\end{array}$ & Extensão \\
\hline $\mathbf{C 1 9}$ & Ginástica para idosos; & Extensão \\
\hline $\mathbf{C 2 0}$ & $\begin{array}{l}\text { Laboratório de Pesquisas e Tecnologias em } \\
\text { Enfermagem, Cuidado em Saúde a Pessoas Idosas - } \\
\text { GESPI } \\
\text { Núcleo de Estudos da Terceira idade - NETI }\end{array}$ & $\begin{array}{l}\text { Pesquisa } \\
\text { Extensão }\end{array}$ \\
\hline
\end{tabular}

Fonte: Dados fornecidos pelos coordenadores de IES participantes. Elaborado pela autora, 2017.

Já o quadro 3 demonstra os principais projetos desenvolvidos especificamente para idosos com deficiência.

Quadro 3 - Projetos desenvolvidos para idosos com deficiência em vinte e dois cursos de graduação na área da saúde em IES da Grande Florianópolis/SC.

\begin{tabular}{|c|c|c|}
\hline Coordenador & Projeto Idoso com Deficiência & Tipo de Projeto \\
\hline C1 & $\begin{array}{l}\text { Educação em saúde visando alcançar o conceito de } \\
\text { práticas corporais como linguagem para mudança de } \\
\text { hábitos e estilo de vida. }\end{array}$ & Não informou \\
\hline $\mathbf{C 2}$ & Atividades de promoção da saúde entre outros. & Não informou \\
\hline \multirow[b]{2}{*}{ C3 } & $\begin{array}{l}\text { Através do Grupo de Estudos da Terceira Idade são } \\
\text { desenvolvidos projetos de caminhada; dança; } \\
\text { ginástica; hidroginástica; musculação; natação; } \\
\text { pilates; Universidade Aberta; Canto; Prevenção e } \\
\text { tratamento das disfunções do assoalho pélvico. }\end{array}$ & Extensão \\
\hline & $\begin{array}{l}\text { Projetos de pesquisa desenvolvidos através do } \\
\text { Laboratório de Gerontologia: Nível de Atividade } \\
\text { Física, Rede de Suporte Social e Funcionalidade } \\
\text { Familiar de Idosos Centenários da Grande } \\
\text { Florianópolis.Programa "Academia da Saúde" em } \\
\text { Santa Catarina: característica e participação de } \\
\text { idosos. Avaliação do equilíbrio e medo de cair em } \\
\text { idosos. }\end{array}$ & Pesquisa \\
\hline C4 & $\begin{array}{l}\text { Projeto de extensão em implante coclear e outras } \\
\text { alterações auditivas com o objetivo de esclarecer } \\
\text { sobre a Política de Atenção à Saúde Auditiva que } \\
\text { oferece próteses auditivas e questões voltadas à } \\
\text { saúde auditiva. }\end{array}$ & Extensão \\
\hline C5 & $\begin{array}{l}\text { Cinesioterapia em pessoas com artrose de joelho, } \\
\text { com objetivo de verificar os efeitos da mesma na } \\
\text { dor e sua melhora; Inclusão digital relacionado com } \\
\text { a demência. }\end{array}$ & Pesquisa \\
\hline
\end{tabular}


Tecnologias desenvolvidas pelas instituições de ensino superior para idosos com deficiência

\begin{tabular}{|c|c|c|}
\hline & $\begin{array}{l}\text { Fisioterapia aquática para idosos com osteoartrose } \\
\text { de coluna e joelho, com objetivo de minimizar a dor } \\
\text { e melhorar a função. }\end{array}$ & Extensão \\
\hline C7 & $\begin{array}{l}\text { Atendimento especializado para portadores de } \\
\text { Parkinson. }\end{array}$ & Não informou \\
\hline C9 & $\begin{array}{l}\text { Afirmou que o curso desenvolve projetos de } \\
\text { pesquisa e extensão, no entanto não especificou. }\end{array}$ & Não informou \\
\hline C10 & Vivendo bem com a Doença de Parkinson & Não informou \\
\hline C16 & Oficina da lembrança & Não informou \\
\hline C19 & $\begin{array}{l}\text { Musculação para Parkinsonianos; Condicionamento } \\
\text { físico para Cardiopatas. }\end{array}$ & Extensão \\
\hline $\mathrm{C} 20$ & $\begin{array}{l}\text { O Programa Grupo de Apoio aos Portadores da } \\
\text { Doença de Parkinson e seus Familiares tem o } \\
\text { objetivo de apoiar os portadores e familiares para } \\
\text { enfrentarem a doença } \\
\text { O Projeto Grupo de Apoio aos Familiares de } \\
\text { Portadores da Doença de Alzheimer oferece espaço } \\
\text { para a partilha de experiências dos portadores e seus } \\
\text { cuidadores, com informação sobre a doença, suas } \\
\text { reivindicações e lutas. }\end{array}$ & Extensão \\
\hline
\end{tabular}

fornecidos pelos coordenadores. Elaborado pela autora, 2017.

\section{Atividades de lazer desenvolvidas pelos cursos de IES para idosos e idosos com deficiência}

A maioria dos coordenadores descreveu que os cursos não desenvolvem atividades de lazer, sendo que 23\% realizam atividades de lazer para idosos e $18 \%$ para idosos com deficiência conforme quadro 4.

Quadro 4 - Atividades de lazer desenvolvidas para pessoas idosas em cinco cursos de graduação na área da saúde em IES da Grande Florianópolis/SC. 
Tecnologias desenvolvidas pelas instituições de ensino superior para idosos com deficiência

\begin{tabular}{|c|l|}
\hline Coordenador & \multicolumn{1}{|c|}{ Atividade de lazer para idoso } \\
\hline C1 & $\begin{array}{l}\text { Desenvolve atividade de lazer, no entanto não especificou e apenas cita } \\
\text { que faz parte do projeto de extensão. }\end{array}$ \\
\hline C2 & $\begin{array}{l}\text { Desenvolve atividade de lazer, no entanto não especificou e apenas cita } \\
\text { que as atividades ocorrem nos estágios desenvolvidos no CATI de São } \\
\text { José. }\end{array}$ \\
\hline C3 & $\begin{array}{l}\text { Desenvolve atividade de lazer, no entanto não especificou e apenas cita } \\
\text { que as atividades ocorrem nas diferentes disciplinas, estágios e projetos } \\
\text { de extensão. }\end{array}$ \\
\hline C6 & $\begin{array}{l}\text { Desenvolve atividade de lazer, tipo prática esportiva para todas as faixas } \\
\text { etárias junto ao Complexo Aquático da Universidade. }\end{array}$ \\
\hline & $\begin{array}{l}\text { Jogos, passeios e viagens, que são desenvolvidos dentro dos projetos de } \\
\text { extensão para idosos. }\end{array}$ \\
\hline
\end{tabular}

Fonte: Dados fornecidos pelos coordenadores. Elaborado pela autora, 2017.

Dos $18 \%$ de coordenadores que descreveram que os cursos desenvolvem atividades de lazer para idosos com deficiência, $50 \%$ especificam tais atividades, conforme apresentado no Quadro 5.

Quadro 5 - Atividades de lazer desenvolvidas para idosos com deficiência em quatro cursos de graduação na área da saúde em IES da Grande Florianópolis/SC.

\begin{tabular}{|c|l|}
\hline Coordenador & \multicolumn{1}{|c|}{ Atividade de lazer para Idoso com Deficiência } \\
\hline C1 & Não descreve atividade, apenas cita que faz parte do projeto de extensão. \\
\hline C2 & $\begin{array}{l}\text { Não descreve atividade, apenas cita que as atividades de lazer ocorrem } \\
\text { nos estágios desenvolvidos no CATI de São José. }\end{array}$ \\
\hline C3 & Dança para os pacientes com Parkinson. \\
\hline C16 & Atividade com computador \\
\hline
\end{tabular}

Fonte: Dados fornecidos pelos coordenadores de IES públicas e privadas, elaborado pelos autores, 2017.

\section{Observações pertinentes}

No instrumento de coleta de dados foi realizada uma pergunta aberta para que os participantes descrevessem alguma observação relacionada ao tema que consideravam importante expressar, além do que já haviam respondido. Essa questão não era obrigatória, no entanto 18,2\% dos participantes expressaram suas opiniões de modo a justificar a falta de atividade/atendimento específicos para pessoas idosas e ou idosos com deficiência; falta de conteúdos sobre rede de atenção e políticas públicas; avaliação de processos formativos e estrutura do curso.

\section{ANÁLISE}

Evidências demonstram problemas relacionados ao acesso aos serviços de saúde e inadequação do modelo de atenção para atender à demanda dos idosos, parecendo existir um 
gargalo no qual especialmente os idosos com ampla carga de morbidades desafiam o sistema que nem sempre consegue atender a essa demanda (MARTINS et al., 2014).

Nesse sentido, destaca-se o papel primordial das IES na formação de profissionais preparados para atender à crescente população de pessoas idosas e com deficiências. Sobretudo, ressalta-se o compromisso social dessas instituições que ao produzir e aplicar tecnologias inovadoras de cuidado implementadas à pessoa idosa com deficiência, além de oferecerem subsídios para formulação e implementação de políticas públicas em saúde, também complementam o atendimento de saúde dos serviços através de suas ações desenvolvidas nas clinicas escola, projetos de extensão e pesquisa, dentre outras.

O Mapa de Ensino Superior no Brasil, nos últimos treze anos demonstrou que o número de IES no país esteve em constante ascensão, com um crescimento total de 102,6\%, sendo 108,2\% nas IES privadas e 71\% nas públicas (CAPELATO et al., 2015). As IES têm a seu favor a produção da tecnologia, que segundo Merhy (2002), mobilizam mais que instrumentos, envolvem o conhecimento de cada profissional ao desenvolver suas habilidades e capacidades em sua área de atuação, qualificando o ensino superior um campo rico na formação de novos profissionais e desenvolvimento de tecnologias, que nessa pesquisa se refere, especialmente, as tecnologias de cuidado.

As IES pesquisadas neste estudo são referentes à mesorregião da Grande FlorianópolisSC e somente em instituições que oferecem cursos de graduação na área da saúde. Com base nos resultados e fundamentado em Minayo (2015), foram criadas duas categorias para discussão, sendo estas: Perfil dos coordenadores de curso; Tecnologias de cuidado dirigidas ao idoso e idoso com deficiência.

No que se refere ao perfil do coordenador de curso, a atual exigência no mercado de trabalho, qualifica-o como gestor universitário, tendo em suas mãos a potencialidade de modelar o curso de graduação e designar melhorias na formação de futuros profissionais com base nas Leis e Diretrizes Nacionais, orientações do Ministério da Educação (MEC), fundamentado em conhecimento científico e apoiado pelo corpo docente qualificado (BASSOLI, 2014).

Segundo o MEC, espera-se que um coordenador de curso participe em reuniões do colegiado da IES, tenha experiência acadêmica e profissional, que sua área de graduação seja pertinente ao curso coordenado, bem como apresente titulação pós-graduação (doutorado, mestrado ou especialização) e realize o regime de trabalho instituído pela IES (BRASIL, 2010c).

De acordo com os dados apresentados pelos participantes desta pesquisa, comparado ao perfil de coordenador almejado pelo MEC, pode-se afirmar que todos os participantes da pesquisa se enquadram no perfil desejado. 
A Política Nacional do Idoso (1994) bem como a Política Nacional de Saúde da Pessoa Idosa (2006) incentivam e apoiam estudo e pesquisa que avaliem e aprimorem a atenção de saúde à pessoa idosa por meio das instituições formadoras, universidades, faculdades e órgãos públicos. Assim como é de interesse nacional fomentar pesquisas em envelhecimento e saúde da pessoa idosa, bem como incentivar a pesquisa e o desenvolvimento tecnológico em todas as áreas do conhecimento relacionadas à pessoa com deficiência, conforme a Lei $n^{0} 7.853 / 1989$, que dispõe sobre apoio à pessoa com deficiência.

Quanto às tecnologias de cuidado destinadas ao idoso e idoso com deficiência, este estudo mostrou que a maioria das IES participantes investe em estudos voltados ao tema do idoso e idoso com deficiência, corroborando ao que se preconiza nas políticas. No entanto, o tema envelhecimento desenvolvido nos currículos (através de estágio supervisionado ou disciplina optativa) e nos projetos de pesquisa, bem como o conhecimento aplicado através de projetos de extensão ainda são pouco abrangentes, considerando a problemática do envelhecimento populacional emergente no Brasil.

As IES participantes demonstraram que há investimentos na produção de conhecimento relacionado à saúde do idoso e idoso com deficiência, através de abordagens em projetos de pesquisa nas áreas de promoção e prevenção a saúde, no estudo ao tema envelhecimento, bem como da funcionalidade familiar do idoso e de seus hábitos e limitações cotidianas. Tais conhecimentos podem ser geradores de tecnologias de cuidado quando testados e aplicados na prática.

Deste modo, percebe-se que as tecnologias de cuidado, vão além do aspecto trabalhointervenção-produção-máquina, podem ser interpretadas como um conjunto de conhecimentos; ferramentas e/ou estratégias que auxiliam na instrumentalização do cuidado, sendo consideradas indispensáveis na prática relacional, interativa e comunicativa prestada em sua maioria nos atendimentos na área da saúde (KOERICH et al., 2006). As tecnologias do cuidado podem ser compreendidas como mediadores da racionalidade e da subjetividade, da intuição, da emoção e das sensações, fazendo da razão e da sensibilidade instrumentos para fortalecer e qualificar o cuidado em saúde (NIETSCHE et al., 2012).

As IES participantes destacaram por meio do desenvolvimento de projetos de extensão e atendimento, tecnologias do cuidado, prestadas ao idoso e idoso com deficiência, sendo agrupadas e destacadas em grandes áreas do conhecimento, como: educação e promoção da saúde; educação permanente; prevenção; tratamento e reabilitação, as quais serão discutidas na sequência. 
A promoção da saúde pode ser considerada uma consequência da educação em saúde, tendo em vista que as origens e concepções da promoção da saúde iniciaram decorrentes de práticas educativas no século XX realizadas por "higienistas" da época. É considerada uma estratégia que proporciona visibilidade e compreensão dos principais fatores de risco e agravos que podem acometer a saúde da população, focando no atendimento ao indivíduo (individual ou coletivo) e elaborando mecanismos com objetivo de reduzir situações de vulnerabilidade e conscientizar a mudança no estilo de vida (JANINI; BESSLER; VARGAS, 2015).

Entre os projetos/atendimentos de destaque na área da promoção e educação em saúde nesta pesquisa, o coordenador C3 chama a atenção para os diferentes projetos que desenvolve sendo alguns deles caminhada, dança, natação para idosos e idosos com deficiência. O coordenador C17 ressalta uma atividade de educação em saúde através do projeto de Nutrição na Terceira Idade (NUTI) com objetivo de trabalhar o estilo de vida saudável; e o coordenador C20 destaca o grupo de apoio mútuo que oferece espaço para partilha de experiências para portadores da Doença de Alzheimer, seus familiares e cuidadores.

A educação permanente, proposta ético-político-pedagógica, instituída pela Portaria n¹98/2004, visa transformar e qualificar a atenção à saúde, por meio da identificação de necessidades de formação e desenvolvimento dos trabalhadores de saúde, construção de estratégias e processos que qualifiquem a atenção e a gestão em saúde, bem como mobilizem a formação de gestores e articule/estimule a transformação das práticas de saúde e educação no âmbito do SUS, nas IES e demais redes de serviços.

No decorrer da descrição de projetos e atendimentos alguns coordenadores destacaram meios e projetos nos quais se desenvolvem atividades de educação permanente com pessoas idosas, onde de modo interdisciplinar e intergeracional, os participantes de um programa trocam experiências populares e conhecimentos científicos em prol do desenvolvimento humano em todas as etapas do ciclo vital, inclusive na velhice.

O coordenador C3 destaca o Grupo de Estudos da Terceira Idade (GETI), o qual proporciona uma Universidade Aberta (UnATI) abrindo espaços para idosos e idosos com deficiência que tem interesse em aprimorar seus conhecimentos em envelhecimento humano.

O Núcleo de Estudos da Terceira Idade (NETI), citado por C20, é um ambiente procurado por pessoas com mais de 50 anos, considerado um polo favorável a educação permanente, o qual desenvolve atividades socioeducativas que favorecem um envelhecimento saudável e propiciam novos sonhos em razão das possibilidades encontradas nesta etapa da vida. O coordenador C14 ressalta que o curso desenvolve um programa de preparação para a aposentadoria, enquadrando-se na proposta de educação permanente. 
A prevenção de saúde, outra tecnologia do cuidado, pode ser classificada em três níveis, sendo que a prevenção primária vai de encontro a promoção da saúde, a qual remove causas e fatores de risco por meio de atividades físicas, imunização entre outras. Já a prevenção secundária, está ligada a ações que detectam um problema de saúde em estágio inicial, mas logo que identificado, realiza o tratamento reduzindo e/ou prevenindo a disseminação ou consequências da doença. E a prevenção terciária, envolve ações que visam reduzir os prejuízos funcionais consequentes a um problema agudo ou crônico já instalado, incluindo medidas de reabilitação (MARTINS, 2016).

Entre os participantes que destacaram projetos relacionados à prevenção encontram-se $O$ coordenador C17 com a proposta de educação continuada a clientes diabéticos; e o coordenador C18 com projeto que avalia o perfil lipídico de idosos e o risco de desenvolver doença coronariana.

A reabilitação, também considerada uma tecnologia do cuidado, é caracterizada como um processo de consolidação de objetivos terapêuticos destinados a auxiliar pessoas com deficiência ou prestes a adquirir deficiências. Os objetivos terapêuticos na reabilitação requerem que a pessoa alcance a funcionalidade ideal seja esta física, sensorial, intelectual, psicológica e social, em seu ambiente fornecendo as ferramentas que necessitam para atingir autonomia e autodeterminação (SANTA CATARINA, 2017).

As atividades de reabilitação citadas na pesquisa estão mais relacionadas ao público idoso com deficiência, entre os projetos citados encontram-se inclusão digital relacionado com a demência, oficina da lembrança, fisioterapia aquática para idosos com osteoartrose de coluna e joelho, musculação para parkinsonianos, condicionamento físico para cardiopatas, grupo de apoio aos portadores da doença de Parkinson e seus familiares, projeto de extensão em implante coclear e outras alterações auditivas.

Evidencia-se a partir disso, que a maior parte dos projetos desenvolvidos para idosos com deficiência engloba os principais tipos de deficiência (físico, intelectual e auditivo), 77\% dos participantes declaravam atender deficiência múltipla, no entanto, não se observou nenhum projeto específico para idosos com acuidade visual diminuída e/ou cegueira, apresentando uma lacuna para a população com déficit visual, e indicando a necessidade de investir mais em projetos voltados a esta área, tendo em vista que uma das principais DCNT que acometem a população idosa está relacionada à diabetes mellitus, e assim como já colocado, uma das consequências desta doença é a retinopatia, a qual pode levar a uma perda parcial ou total da visão. 
No que se refere ao atendimento de saúde ao idoso com deficiência, a pesquisa mostrou que $54 \%$ de idosos na faixa etária entre 60 a 65 anos procuram o serviço de saúde e educacional em busca de atendimentos e programas que o permitam melhorar sua condição de vida e saúde.

Diante deste fato, evidencia-se que o idoso jovem é quem mais busca o serviço, e ressaltase a importância das IES continuarem desenvolvendo projetos e atendimentos voltados a este público, entretanto vale lembrar o crescente número de idosos longevos e centenários que requerem, igualmente, tecnologias de cuidado que deem conta das suas especificidades. Finalizase reconhecendo que, embora as IES não tenham foco assistencial, mediante a maioria dos projetos demonstrados, desempenham importante papel na atenção à saúde da pessoa idosa e idosa com deficiência.

\section{CONSIDERAÇÕES FINAIS}

A pesquisa conseguiu identificar as principais tecnologias inovadoras de cuidado implementadas ao idoso e idoso com deficiência junto as IES da Grande Florianópolis/SC que oferecem cursos na área da saúde.

Considera-se que, mesmo diante dos resultados alcançados, a pesquisa poderia ter maior amplitude de conhecimento relacionado aos atendimentos e projetos realizados ao público idoso e idoso com deficiência, no entanto muitos participantes não informaram e/ou duplicaram a informação quanto aos atendimentos e projetos desenvolvidos ao público em questão, prejudicando de certa forma a análise desta pesquisa.

Quanto a isso, vale a reflexão, de que muitas vezes, devido à construção cultural do país, pensa-se que o envelhecimento é sinônimo de senilidade, sendo assim os participantes acabaram generalizando seus projetos e atendimentos aos dois públicos, o idoso senescente com o idoso que apresenta alguma deficiência.

Sugere-se que as IES invistam mais nos currículos de cursos de graduação da área da saúde com relação a conhecimentos a respeito dos direitos e deveres dos idosos e idosos com deficiência, para que os futuros profissionais possam sair mais capacitados para atender a esta demanda populacional e, assim venham cumprir e colaborar com propostas e políticas do governo.

\section{REFERÊNCIAS}


BASSOLI, Dyjalma Antonio. O coordenador de curso superior de instituições de ensino privadas: análise dos contributos políticos, gerenciais, acadêmicos e institucionais. 2014. 239 f. Tese (Doutorado) - Curso de Engenharia de Produção, Departamento de Engenharia de Produção, Universidade de São Paulo, São Carlos, 2014. Disponível em: <http://www.teses.usp.br/teses/disponiveis/18/18157/tde-17072014-092602/en.php>. Acesso em: 21 maio 2017.

BRASIL. Ministério da Saúde. Secretaria de Atenção à Saúde. Atenção à saúde da pessoa idosa e envelhecimento. Brasília: Série B. Textos Básicos de Saúde, 2010a. 46 p. Disponível em: <http://bvsms.saude.gov.br/>. Acesso em: 5 maio 2017.

- Ministério da Saúde. Secretaria de Atenção à Saúde. Política Nacional de Saúde da Pessoa com Deficiência. Brasília: MS, 2010b. 21 p. Disponível em: $<$ http://bvsms.saude.gov.br>. Acesso em: 5 maio 2017.

- Ministério da educação. Instrumento de Avaliação de Cursos de Graduação: Bacharelado e Licenciatura. Brasília: Ministério da Educação, 2010c. 21 p. Disponível em: $<$ http://download.inep.gov.br/download/superior/condicoesdeensino/2010/instrumento_reco nhecimento_bacharelado_licenciatura3.pdf>. Acesso em: 21 maio 2017.

Departamento de atenção básica. Rede de Cuidado à Pessoa com Deficiência. 2012a. Disponível em: <http://dab.saude.gov.br/>. Acesso em: 3 maio 2017.

.Secretaria especial dos direitos da pessoa com deficiência. Viver sem Limite. Disponível em: <http://www.pessoacomdeficiencia.gov.br/app/viver-sem-limite>. Acesso em: 07 maio 2017.

CAPELATO, Rodrigo et al (Org.). Mapa do Ensino Superior no Brasil. 5. ed. São Paulo: Assessoria Econômica do Semesp, 2015. 212 p. Disponível em:

$<$ http://convergenciacom.net/pdf/mapa-ensino-superior-brasil-2015.pdf > . Acesso em: 20 maio 2017.

GIRONDI, Juliana Balbinot Reis et al. A rede de atenção a saúde e de suporte social à pessoa idosa com deficiência na grande Florianópolis e as tecnologias de cuidado. Projeto Fapesc n ${ }^{\circ}$ 07/2013. Universidade Federal de Santa Catarina (UFSC); Núcleo de Estudos da Terceira Idade (NETI/Florianópolis/SC), Florianópolis, SC, 2013, 63 p.

JANINI, Janaina Pinto; BESSLER, Danielle; VARGAS, Alessandra Barreto de. Educação em saúde e promoção da saúde: impacto na qualidade de vida do idoso. Saúde Debate, Rio de Janeiro, v. 39, n. 105, p.480-490, jun. 2015. Disponível em: <http://www.scielo.br/pdf/sdeb/v39n105/0103-1104-sdeb-39-105-00480.pdf>. Acesso em: 23 maio 2017.

KOERICH, Magda Santos et al. Tecnologias de cuidado em saúde e enfermagem e suas perspectivas filosóficas. Texto e Contexto, Florianópolis, v. 15, n. 22, p.178-185, out. 2006. Disponível em: <http://www.scielo.br/pdf/tce/v15nspe/v15nspea22.pdf>. Acesso em: 23 maio 2017.

MARTINS, Aline Blayaet al. Atenção Primária a Saúde voltada as necessidades dos idosos: da teoria à prática. Ciência \& Saúde Coletiva, [s.l.], v. 19, n. 8, p.3403-3416, ago. 2014. FapUNIFESP (SciELO). http://dx.doi.org/10.1590/1413-81232014198.13312013. 
Tecnologias desenvolvidas pelas instituições de ensino superior para idosos com deficiência

MARTINS, Leonam. Prevenção de doenças e promoção da saúde na terceira idade. 2016.

Disponível em: < http://sbgg.org.br/prevencao-de-doencas-e-promocao-da-saude-na-terceiraidade/>. Acesso em: 23 maio 2017.

MERHY, E. E. et al. Em busca de ferramentas analisadoras das tecnologias em saúde: a informação e o dia a dia de um serviço, interrogando e gerindo trabalho m saúde. In Merhy, E. E. eOnocko R. (org.) Agir em saúde. Um desafio para o público, 2ª ed. São Paulo: UCITEC, 2002.

MINAYO, Maria Cecília de Souza (Org.). Pesquisa Social: Teoria, método e criatividade. 34. ed. Petrópolis: Vozes, 2015. 108 p.

NIETSCHE, Elisabeta Albertina et al. Tecnologias inovadoras do cuidado em enfermagem. Revista de Enfermagem da Ufsm, Santa Maria, v. 2, n. 1, p.182-189, abr. 2012. Disponível em: <periodicos.ufsm.br>. Acesso em: 05 jul. 2016.

RESENDE, M.C.; NERI, A.L. Envelhecer com deficiência física: possibilidades e limitações. IN: NERI, A.L. (ORG). Qualidade de vida na velhice: enfoque multidisciplinar. Campinas: Alínea, 2011. p. 231-250.

SANTA CATARINA. Governo do Estado de Santa Catarina. Secretaria Estadual de Saúde. O que é reabilitação. Disponível em:

$<$ http://portalses.saude.sc.gov.br/index.php?option $=$ com_content\&view $=$ article\&id $=2165$ :oque-e-reabilitacao\&catid=813\&Itemid=448> . Acesso em: 23 maio 2017. 